\title{
Análisis económico de productores y comercializadores de nopal en el Valle de Teotihuacán
}

\author{
Economic Analysis of Nopal Production and Marketing in Teotihuacan Valley
}

Análise econômica de produtores e comerciantes de nopal no vale do Teotihuacán

Blanca Estela Hernández Bonilla

Universidad Autónoma del Estado de México, CU UAEM Valle de Teotihuacán, México. behernandezb@uaemex.mx https://orcid.org/0000-0003-0925-7286

Adriana Mercedes Ruiz Reynoso Universidad Autónoma del Estado de México, CU UAEM Valle de México, México. amruizr@uaemex.mx https://orcid.org/0000-0003-4294-2912

Verónica Ramírez Cortés

Universidad Autónoma del Estado de México, CU UAEM Valle de Teotihuacán, México.

vramirezc@uaemex.mx https://orcid.org/0000-0001-6541-6769

Sendy Janet Sandoval Trujillo Universidad Autónoma del Estado de México, CU UAEM Valle de Teotihuacán, México. sjsaldovalt@uaemex.mx https://orcid.org/0000-0001-6966-7135

Margarita Dávila Hernández Tecnológico de Estudios Superiores de Coacalco, México. davilahernandezmargarita@gmail.com https://orcid.org/0000-0002-2361-9522 


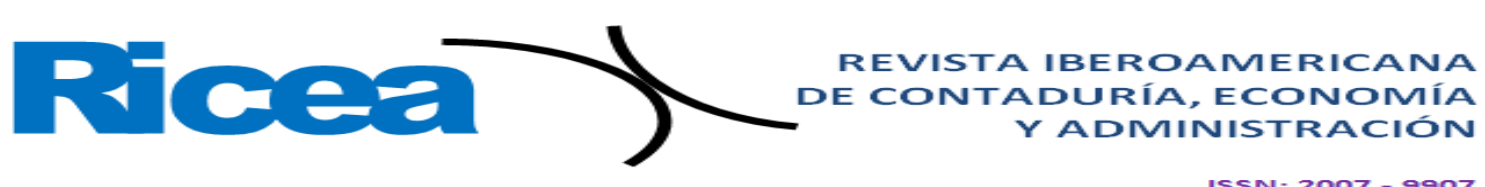

esqueleto sorprendentemente duro, lo que, aunado a su gran tamaño, les ha permitido resistir a las heladas, al fuego, y a los ataques de los campesinos cuando intentaban eliminarlos para preparar terrenos para la ganadería (ver figura 1).

Figura 1. Plantación de nopal en el Estado de México.

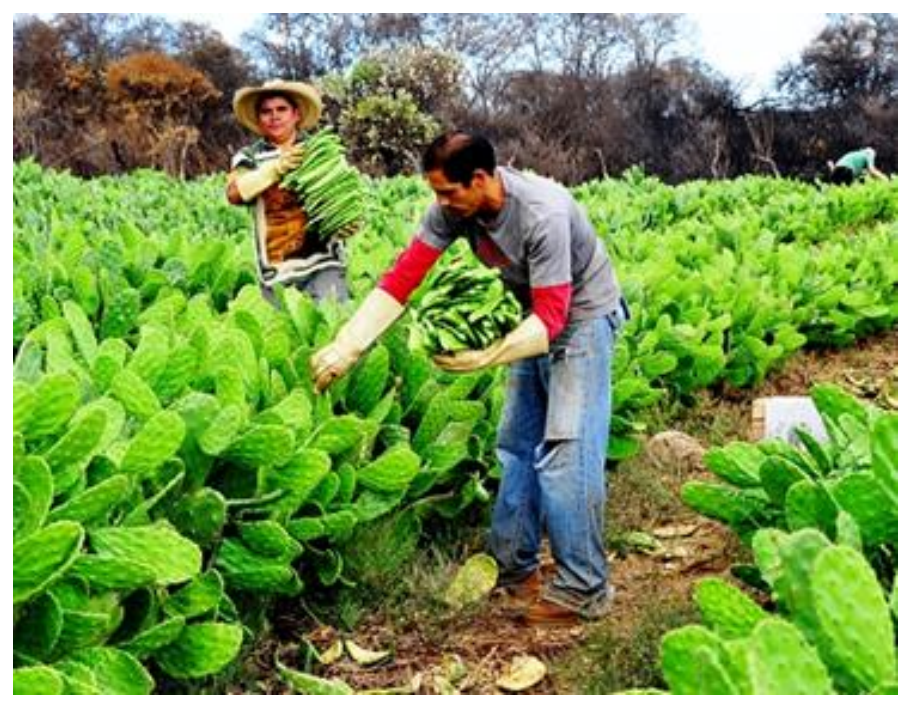

Fuente: Coordinadora Nacional de las Fundaciones Produce (Cofupro, 2018).

A los nopales que han sido domesticados con mayor intensidad se les llama dóciles. Las tendencias principales de selección en la domesticación tienen que ver con criterios de utilidad y gustos de los campesinos. Así, para el consumo de nopalitos se buscan pencas jóvenes y delgadas, de poca baba, fibra y espina, y que se oxiden lentamente al ser cortadas.

En las tunas se seleccionan las grandes y dulces, con abundante y jugosa pulpa, semillas pequeñas y no numerosas, cáscara delgada sin espinas y pocos ahuates; el rojo predomina, pero hay otros colores.

De acuerdo con información de la Conabio (2014), iniciada la década de 1950, las plantaciones comerciales para nopalitos y tunas llegaron a cubrir 80000 hectáreas, pero en los años 80 sufrieron una drástica reducción debido a plagas, susceptibilidad por excesiva homogeneidad genética y mala elección de suelos, así como a factores climáticos externos. Hoy existen cerca de 56000 hectáreas cultivadas, de las cuales $78 \%$ se dedican a la producción de tuna, cerca de $20 \%$ a la de nopalito y menos de $2 \%$ a la de xoconostle (Conabio, 2014). 


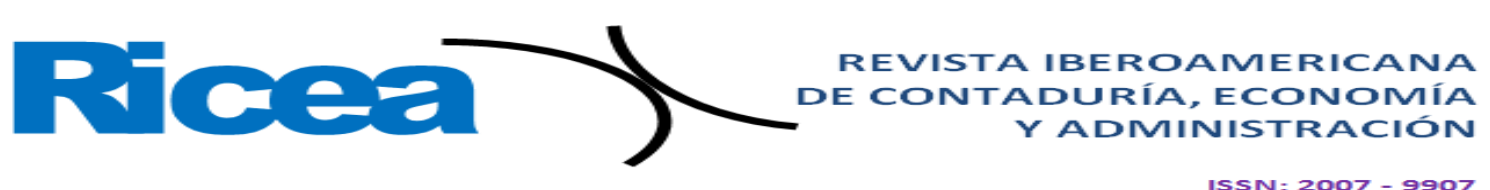

La oferta y la demanda del nopal aumentan día a día. Tanto en el aprovechamiento de poblaciones silvestres como en los cultivos intensivos se requieren calendarios y prácticas de propagación, plantación, poda, fertilización, manejo de plagas y cosecha que optimicen la producción con métodos que no dañen al producto fresco y que garanticen la madurez adecuada del mismo. Las nopaleras silvestres actualmente cubren cerca de un millón y medio de hectáreas, principalmente en el Altiplano y el Bajío. En ellas se recolectan nopalitos, tunas y xoconostles, tanto para autoconsumo como para venta en fresco o procesados (Conabio, 2014).

\section{Marco legal}

En cuanto a las nopaleras silvestres, aplica la Ley General de Vida Silvestre y la Norma Oficial Mexicana de Protección a Especies Nativas de México de Flora y Fauna Silvestre. En la comercialización internacional, las cactáceas son reguladas por la Convención sobre el Comercio Internacional de Especies Amenazadas de Fauna y Flora Silvestres (Conabio, 2014).

Respecto a los recursos fitogenéticos para la agricultura y la alimentación cada vez toma importancia y es un factor integral para la biodiversidad agrícola, este recurso es esencial para fomentar un desarrollo sostenible, para esto se cuenta con la Ley Federal de Producción, Certificación y Comercio de Semillas (Gaceta del Senado, 2004). En la comercialización, el uso de signos distintivos está regulado en el ámbito internacional por los Acuerdos sobre Derechos de Propiedad Intelectual y Comercio en la Organización Mundial de Comercio; y en México, por la Ley de la Propiedad Industrial (OMPI, 2018).

En los huertos o nopaleras de solar, la producción obedece, en cantidad y variedad, al consumo familiar y a la venta en los mercados locales y regionales, lo que hace del nopal y sus derivados un importante recurso para las poblaciones locales. El cultivo en plantaciones comenzó en la década de 1950 y hoy existen cerca de 60,000 hectáreas cultivadas, de las cuales 78\% se dedican a la producción de tuna, cerca de $20 \%$ a la de nopalito y menos de $2 \%$ a la de xoconostle (Cometuna, Red Nopal y Conabio 2009). 


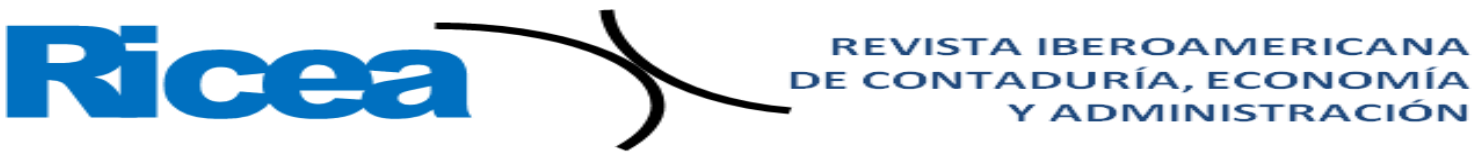

$15 S N=2007-9907$

Tabla 1. Datos estadísticos de la Región del Valle de Teotihuacán.

\begin{tabular}{|l|r|r|}
\hline \multicolumn{1}{|c|}{ Municipio } & \multicolumn{1}{|c|}{ Superficie $\mathbf{( k m}^{\mathbf{2}} \mathbf{~}$} & Población total \\
\hline Acolman & 83.86 & 136558 \\
\hline Axapusco & 231.37 & 25559 \\
\hline Nopaltepec & 84 & 8895 \\
\hline Otumba & 195.72 & 34232 \\
\hline San Martín de las Pirámides & 67.32 & 24851 \\
\hline Temascalapa & 164.61 & 35987 \\
\hline Teotihuacán & 83.18 & 53010 \\
\hline Total & $\mathbf{9 1 0 . 0 6}$ & $\mathbf{3 1 9 0 9 2}$ \\
\hline
\end{tabular}

Fuente: Elaboración propia con base en Inegi (2010).

Dentro de las actividades agrícolas que se desarrollan en la región, se destaca el cultivo y cosecha del nopal (tunero y verdura). Según datos del Servicio de Información Agroalimentaria y Pesquera [SIAP] (2016), el Estado de México ocupa el primer lugar en la producción del nopal tunero a nivel nacional; el distrito de Zumpango es el que mayor superficie sembrada tiene. Este distrito, cabe señalar, se integra por los municipios de Acolman, Axapusco, Nopaltepec, Otumba, San Martín de las Pirámides, Temascalapa, Teotihuacán y Tlalnepantla de Baz. De estos, el municipio de San Martín de las Pirámides genera la mayor producción, seguido de Otumba, Nopaltepec, Axapusco y Teotihuacán. Sin considerar el municipio de Acolman, en el Valle de Teotihuacán alrededor de $27.5 \%$ de la superficie territorial se encuentra sembrada con nopal, sea verdura o tunero, lo cual destaca la relevancia de la actividad en cuestión en la región (SIAP, 2016). Por su parte, en la tabla 2 se muestran estos datos estadísticos.

Es importante destacar el caso del municipio de San Martín de las Pirámides: 70 \% de su territorio se encuentra sembrado de nopal, principalmente tunero, por lo que dicha actividad es muy importante para sus pobladores. 


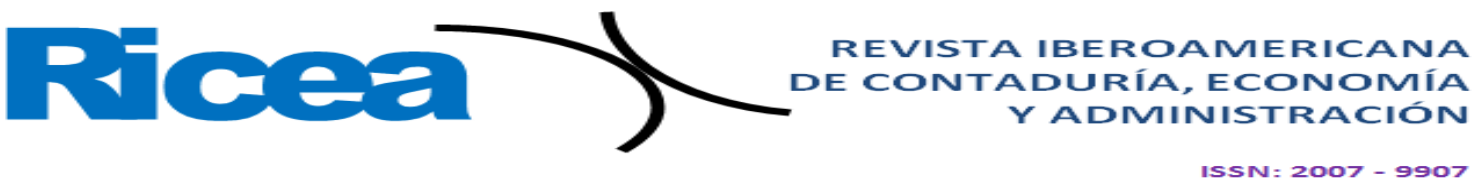

Tabla 2. Superficie sembrada de nopal en los municipios del Valle de Teotihuacán

\begin{tabular}{|l|r|l|r|l|r|}
\hline Municipio & $\begin{array}{l}\text { Superficie } \\
\text { territorial } \\
\text { en (ha) }\end{array}$ & $\begin{array}{l}\text { Superficie } \\
\text { sembrada } \\
\text { Nopalitos } \\
\text { (ha) }\end{array}$ & $\begin{array}{l}\text { Superficie } \\
\text { sembrada } \\
\text { Tuna (ha) }\end{array}$ & $\begin{array}{l}\text { Total de } \\
\text { superficie } \\
\text { sembrada } \\
\text { Nopal }\end{array}$ & $\begin{array}{l}\text { Superficie } \\
\text { territorial } \\
\text { sembrada de } \\
\text { nopal }\end{array}$ \\
\hline Axapusco & 23137 & 14 & 3102 & 3116 & $13.5 \%$ \\
\hline Nopaltepec & 8400 & 0 & 2890 & 2890 & $34.4 \%$ \\
\hline Otumba & 19572 & 446 & 3283 & 3729 & $19.1 \%$ \\
\hline $\begin{array}{l}\text { San Martín de } \\
\text { las Pirámides }\end{array}$ & 6732 & 101 & 4633 & 4734 & $70.3 \%$ \\
\hline Temascalapa & 16461 & 0 & 1498 & 1,498 & $9.1 \%$ \\
\hline Teotihuacán & 8318 & 3 & 1556 & 1,559 & $18.7 \%$ \\
\hline Total & 82620 & 564 & 16962 & 17526 & $27.5 \%$ \\
\hline
\end{tabular}

Fuente: Elaboración propia con base en SIAP (2016).

\section{Estado del arte del nopal}

El nopal es una cactácea originaria del continente americano. Se han detectado unas 215 especies, de las cuales en México hay 100 especies del género Opuntia, llamado así por sus características, a saber: planta arborescente, arbustiva, espinosa, con ramas desde la base, flores hermafroditas, fruto en baya, entre otras. Esta planta ocupa un lugar preponderante en la cultura mexicana, tanto por su presencia en la vegetación como por la cantidad de usos que se la da y por ser un ícono de la identidad mexicana al formar parte del escudo nacional: es sobre un nopal donde posa el águila, señal que determinó el lugar de asentamiento de Tenochtitlán, que significa 'lugar del nopal' o 'tunas sobre la piedra' (Conabio, 2014).

Diversos autores citados por Galicia et al. (2017) consideran que son amplios los beneficios que aporta a la salud el consumo diario de nopal, entre algunos de estos están:

- $\quad$ Es fuente de manganeso y fósforo en la dieta, lo cual contribuye al desarrollo adecuado de los huesos y mantiene alerta al sistema inmunológico.

- $\quad$ Proporciona fibra digestiva, lo cual permite al organismo tener una sensación de satisfecho y reduce el apetito, controlando así el peso y la obesidad, además de contribuir a la digestión mediante el trabajo adecuado del intestino: acelera el paso de los alimentos, favorece la absorción de nutrientes, ayuda a disminuir la celulitis y a retener líquidos. 


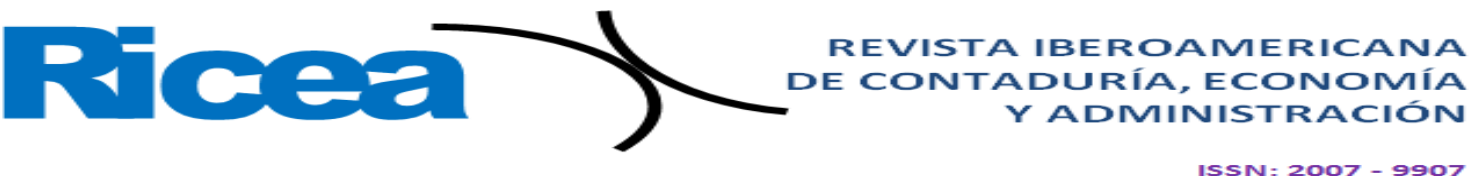

- $\quad$ Es fuente de alrededor de $13 \%$ del valor diario de minerales requeridos por el organismo, ya que contiene calcio, magnesio, sodio, hierro y potasio, lo cual ayuda a eliminar toxinas dañinas en el organismo, desintoxicándolo y apoyando el funcionamiento del hígado.

- $\quad$ Por su contenido de vitaminas A, C, B1, B2 y B3, ayuda al control de la diabetes y la hiperglucemia, reduciendo los niveles altos de azúcar en la sangre, así como las concentraciones de glucosa, colesterol y triglicéridos.

Por otro lado, Mandujano, Morales, Herrera, Corona y Juárez (2018) reportan en sus investigaciones que el uso del nopal tiene propiedades antioxidantes, al disminuir la velocidad del deterioro por corrosión (oxidación) en muestras de acero AISI 1018, 1045 y 4140 expuestas a una mezcla acuosa de ácido sulfúrico $\left(\mathrm{H}_{2} \mathrm{SO}_{4}\right)$ con extracto de nopal, con una eficiencia de inhibición de $95 \%$.

Algunas de las características de calidad buscadas por los consumidores y establecidas en la norma oficial mexicana NMX-FF-068-SCFI-2006 (Secretaría de Economía, 2015) son: el tamaño, la frescura, libres de pudrición, enteros, bien formados, con coloración, sabor y olor propios de la especie (Maki et al., 2015).

En México se encuentra principalmente en regiones desérticas y frías. Según datos del SIAP (2016), la superficie sembrada de nopal (incluye forrajero, verdura y tunero) a diciembre del 2016 a nivel nacional era de 77592 hectáreas, de las que se obtuvo una producción de 1453895 toneladas de producto, lo que dejó un ingreso de 3504994.23 pesos. Es importante destacar que la mayor parte de la superficie sembrada es de nopal tunero, 47632 ha, sin embargo, la mayor producción se obtiene del nopal verdura, 810939 toneladas (ver tabla 3).

Tabla 3. Producción de nopal en México

\begin{tabular}{|l|r|r|r|r|}
\hline Nopal & $\begin{array}{l}\text { Superficie } \\
\text { sembrada } \\
\text { (hectáreas) }\end{array}$ & $\begin{array}{l}\text { Superficie } \\
\text { cosechada } \\
\text { (hectáreas) }\end{array}$ & $\begin{array}{l}\text { Producción } \\
\text { (toneladas) }\end{array}$ & $\begin{array}{l}\text { Valor } \\
\text { producción } \\
\text { (miles de pesos) }\end{array}$ \\
\hline Forrajero & 17340 & 15623 & 180251 & 80392.28 \\
\hline Verdura & 12620 & 12227 & 810939 & 2062714.20 \\
\hline Tuna & 47632 & 45399 & 462705 & 1361887.75 \\
\hline Totales & 77592 & 73249 & 1453895 & 3504994.23 \\
\hline
\end{tabular}

Fuente: Elaboración propia con base en SIAP (2016). 


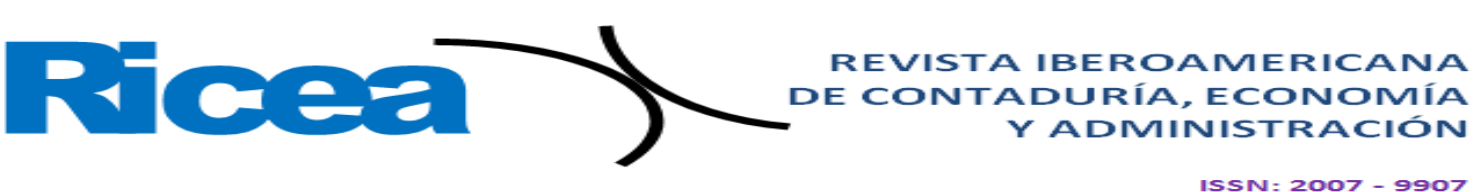

De acuerdo con la clasificación del SIAP (2016), por nopal debe entenderse el forrajero, es decir, el que se utiliza como alimento para los animales; en cuanto a los nopalitos, existen diversos tipos o variedades: el verdura (tradicional), el de invernadero, de macrotúnel y orgánico; por su parte, la tuna puede ser del tipo: alfajayucan, amarilla, blanca burrón, blanca cristalina, criolla, pico chulo, roja y xoconostle.

\section{Producción del nopal en el Valle de Teotihuacán}

En el Estado de México hay 950 hectáreas sembradas de nopal verdura en siete distritos; el distrito de Zumpango es el que registra el mayor número de hectáreas sembradas, 572 ha, lo que representa $60 \%$ de la producción. En la figura 2 se observa la cantidad de hectáreas sembradas en cada distrito. Por su parte, en la figura 3, se puede observar la cantidad de hectáreas sembradas de nopal verdura en siete municipios del distrito de Zumpango dedicados a ello.

Figura 2. Número de hectáreas sembradas de nopal en el Estado de México.

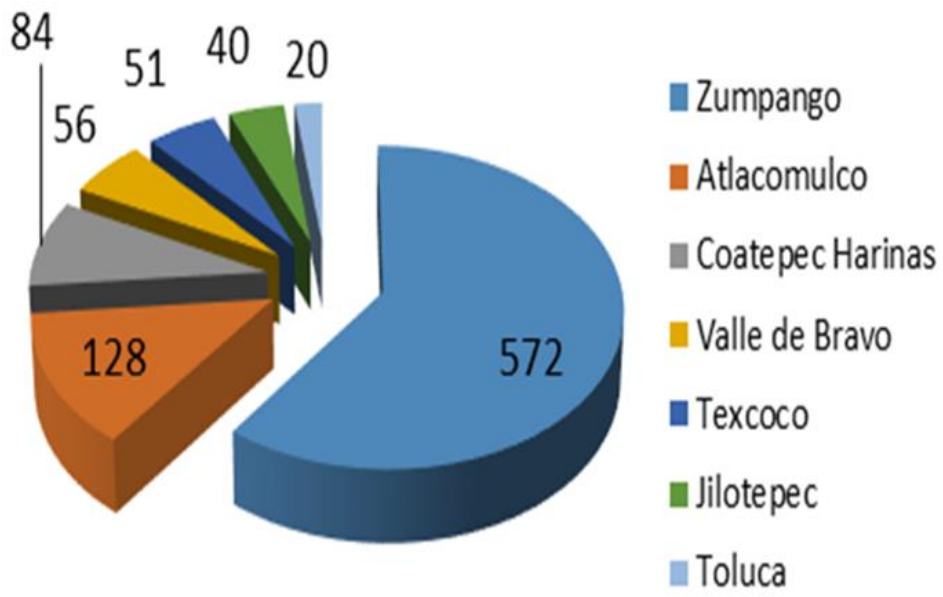

Fuente: Elaboración propia con base en SIAP (2016). 
Figura 3. Número de hectáreas sembradas de nopal en el distrito de Zumpango.

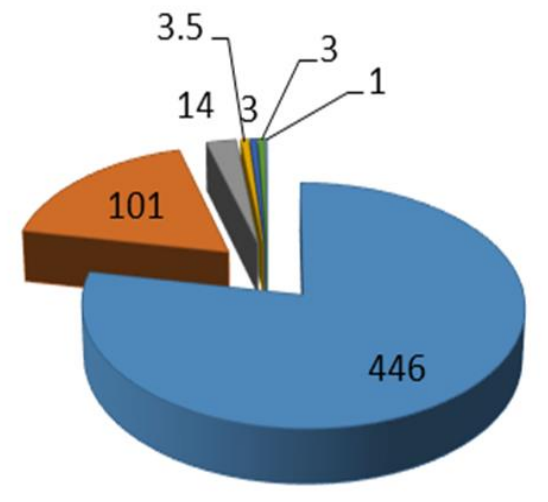

\author{
๑Otumba \\ $\square$ San Martín de \\ las Pirámides \\ $\square$ Axapusco \\ $\square$ Melchor \\ Ocampo \\ घCuautitlán \\ Izcalli \\ घTeotihuacán
}

$\square$ Tultepec

Fuente: Elaboración propia con base en SIAP (2016).

Tal como se observa en la figura 3, el municipio de Otumba es el principal productor de nopalitos: $78 \%$ de las hectáreas sembradas de nopal verdura están en este municipio, particularmente en el poblado de Cuautlacingo, donde desde hace unos 20 años comenzaron a cambiar los sembradíos de tuna por nopal verdura (actualmente todos los agricultores de la zona se dedican a la producción del nopal verdura). Respecto a la producción de tuna, el Estado de México es el principal productor con 17115 hectáreas. Allí, la producción se desarrolla solo en dos distritos: el de Texcoco y el de Zumpango. En el primero se tiene únicamente 19 hectáreas sembradas; mientras que, en el segundo, 17096 hectáreas, la mayoría de las cuales se encuentra en la región del Valle de Teotihuacán, donde San Martín de las Pirámides es el principal productor, seguido de Otumba y Axapusco (ver figura 4). 


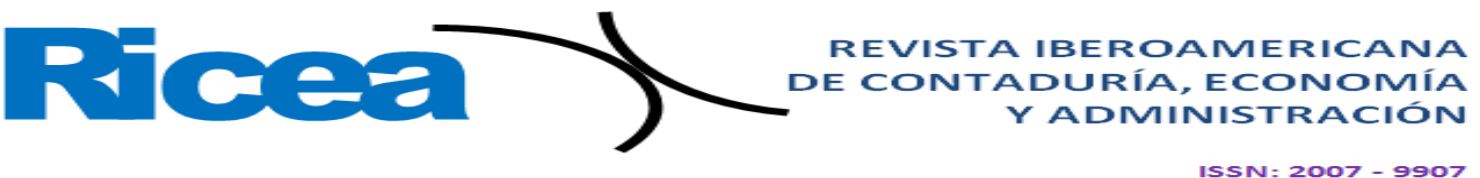

Figura 4. Total de hectáreas sembradas de tuna en el distrito de Zumpango, Estado de México.

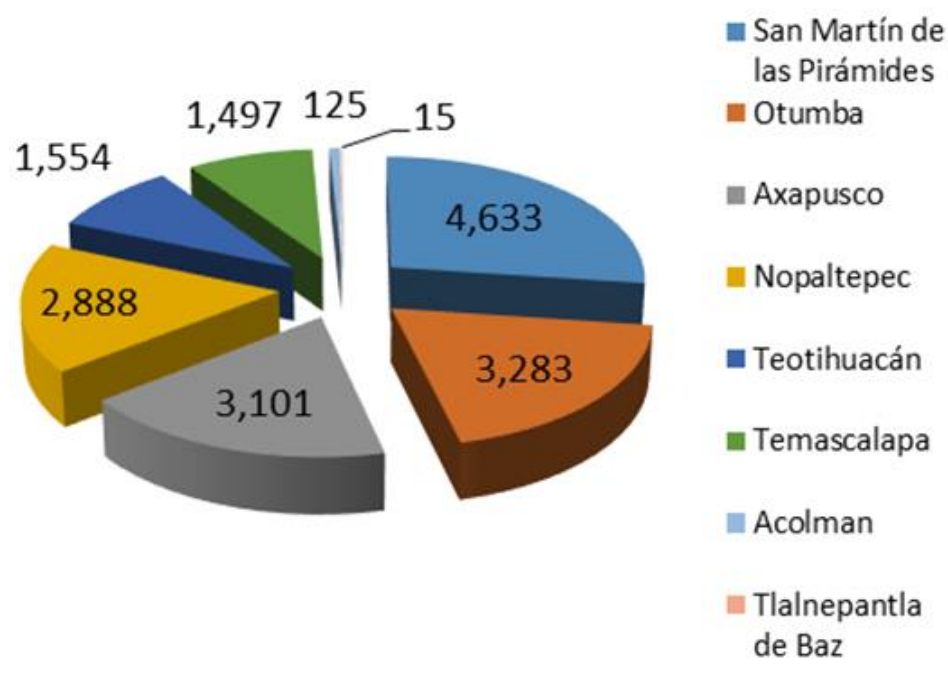

Fuente: Elaboración propia con base en SIAP (2016).

La derrama económica que deja la producción de ambos productos en el Valle de Teotihuacán es de 498769.42 pesos (ver tabla 4).

Tabla 4. Producción de nopal en el Valle de Teotihuacán.

\begin{tabular}{|c|c|c|c|c|}
\hline Nopal & $\begin{array}{l}\text { Superficie } \\
\text { sembrada } \\
\text { (hectáreas) }\end{array}$ & $\begin{array}{l}\text { Superficie } \\
\text { cosechada } \\
\text { (hectáreas) }\end{array}$ & $\begin{array}{l}\text { Producción } \\
\text { (toneladas) }\end{array}$ & $\begin{array}{l}\text { Valor } \\
\text { Producción } \\
\text { (miles de } \\
\text { pesos) }\end{array}$ \\
\hline Verdura & 564 & 564 & 70305 & 70615.92 \\
\hline Tuna & 17081 & 17081 & 190275 & 428153.50 \\
\hline Totales & 17645 & 17645 & 260580 & 498769.42 \\
\hline
\end{tabular}

Fuente: Elaboración propia con base en SIAP (2016).

Un análisis de los datos de la tabla 4 revela que la producción de tuna es la más importante en la región, sin embargo, no se puede dejar de lado la producción del nopal verdura, ya que en una superficie de alrededor de $3 \%$ en comparación con la superficie sembrada de tuna deja una derrama económica de $16 \%$ en relación con esta última. Quizá esta sea la razón por la que en los últimos años algunos productores han dejado de sembrar tuna para sembrar nopal verdura. 


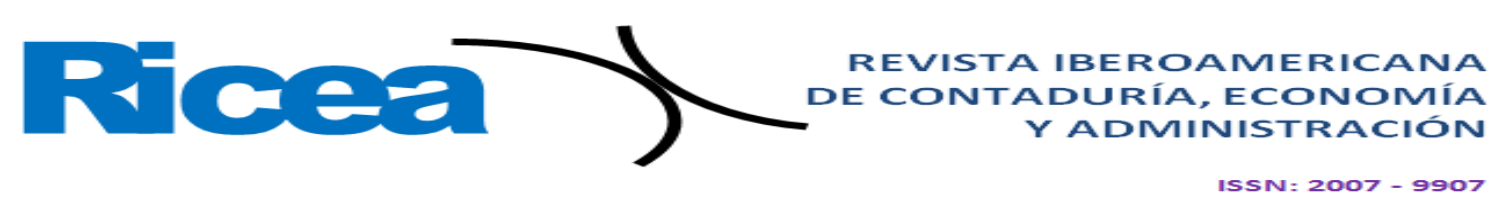

despunta se corre el riesgo de que se eche a perder pronto (en un periodo de dos o tres días), lo mismo sucede cuando se corta la fruta con gancho.

Una vez cortado el fruto se debe limpiar para eliminar las espinas y quitar la humedad del rocío. Si este proceso se hace de forma manual, se realiza en una zona alejada de las huertas, en las afueras, llamada era, donde se barren los frutos con escobas de cambray hasta dejarlos libres de espinas. Entonces se empacan en cajas, generalmente huacales de madera; hay una clasificación de primera, segunda o tercera calidad en función del tamaño de la fruta.

Actualmente, existen máquinas desespinadoras y desahuatadoras que son recomendables porque maltratan menos el fruto y, como se hace bajo la sombra, se prolonga su lozanía. En la región del Valle de Teotihuacán hay productores que han adquirido este tipo de maquinaria y quienes no la tienen pagan por que les realicen este proceso (Aguilar, 2003).

En el Valle de Teotihuacán la comercialización se realiza en tres tianguis de la zona, uno ubicado en el municipio de San Martín de las Pirámides, otro en el municipio de Otumba y uno más en el municipio de Nopaltepec. A estos tianguis acuden los productores para realizar la venta de la fruta, así como compradores con camiones grandes, quienes se llevan la fruta a otros lugares. Allí el precio es de acuerdo con la oferta y demanda, normalmente en ventas de mayoreo y de contado. Otros productores llevan su producto a centrales de abastos cercanas, centros comerciales, lo venden al menudeo sobre las carreteras o alrededor de la zona arqueológica y hay quienes la exportan.

\section{El valor compartido, la brecha a una nueva visión empresarial}

En el tiempo actual, los empresarios se enfrentan a un cambio de paradigmas, y para poder hacerle frente se requiere redefinir el propósito de las empresas, debido a que de ello depende su existencia y progreso (Porter y Kramer, 2006).

El modelo original puede ser completado con la adición de una sexta fuerza que es fundamental y no debe ser excluida: el poder público (Gobierno), el cual se considera como un factor que analiza las medidas de la toma de decisión dentro de la industria. Es importante destacar que la creación del valor empieza por la ventaja competitiva que pudiera existir. Tener una noción sobre la asignación y utilización de los recursos a lo largo de la cadena quizá sea la opción que permita un mejor rendimiento y una mayor eficacia. 


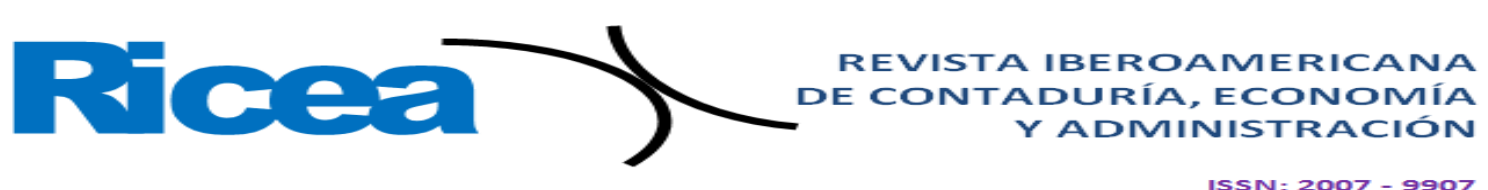

Figura 5. Conceptos principales como competitividad y estrategia.

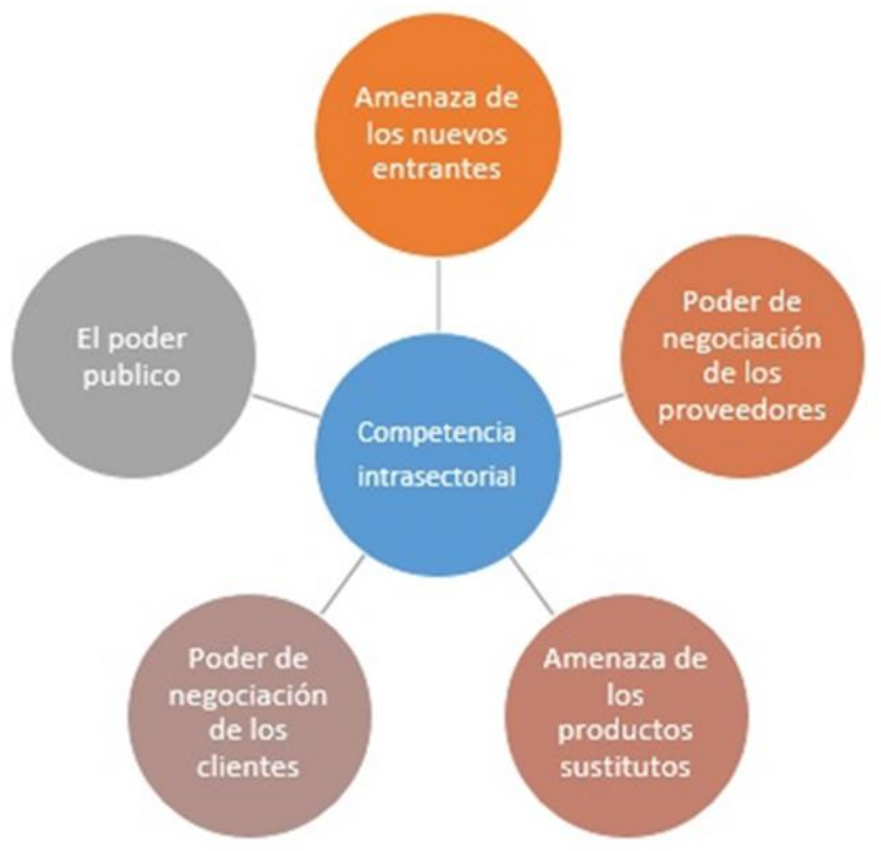

Fuente: Porter y Kramer (2006).

Derivado de un estudio enfocado en industrias y empresas exitosas, Michael E. Porter y Mark R. Kramer (2006) hicieron una selección y desarrollo de elementos necesarios para que las organizaciones se conviertan en competitivas, a partir de lo cual extrajeron las cuatro fuentes de la ventaja competitiva, en su conjunto denominadas el diamante de la competitividad (ver figura 6).

Porter y Kramer (2006), en su publicación Estrategia y sociedad, trazan un vínculo entre la responsabilidad social corporativa (RSC) y la ventaja competitiva, donde se mencionan los principios del concepto de valor compartido, el cual tiene como impacto la innovación y crecimiento empresarial. Derivado de lo anterior, Porter y Kramer (2006) definen estrategia empresarial como la búsqueda deliberada de un plan de acción que desarrolle la ventaja competitiva de una empresa que logre el crecimiento y expansión del mercado reduciendo su competencia. El valor compartido, según Porter y Kramer (2011), establece metodologías para la creación de nuevos modelos y formas de ganar dinero que tengan como interés principal la situación actual de la sociedad. 


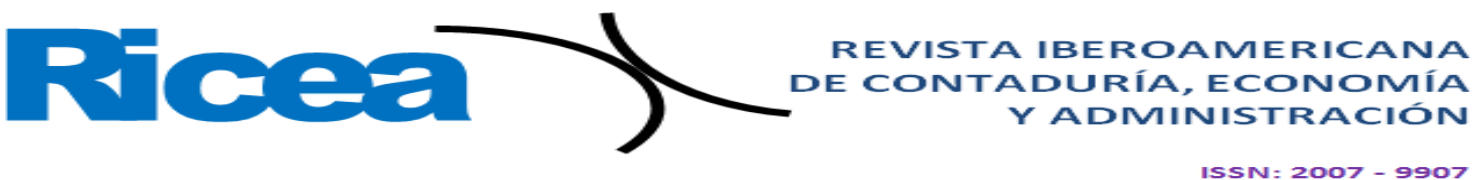

Figura 6. Factores para el diamante de la competitividad.

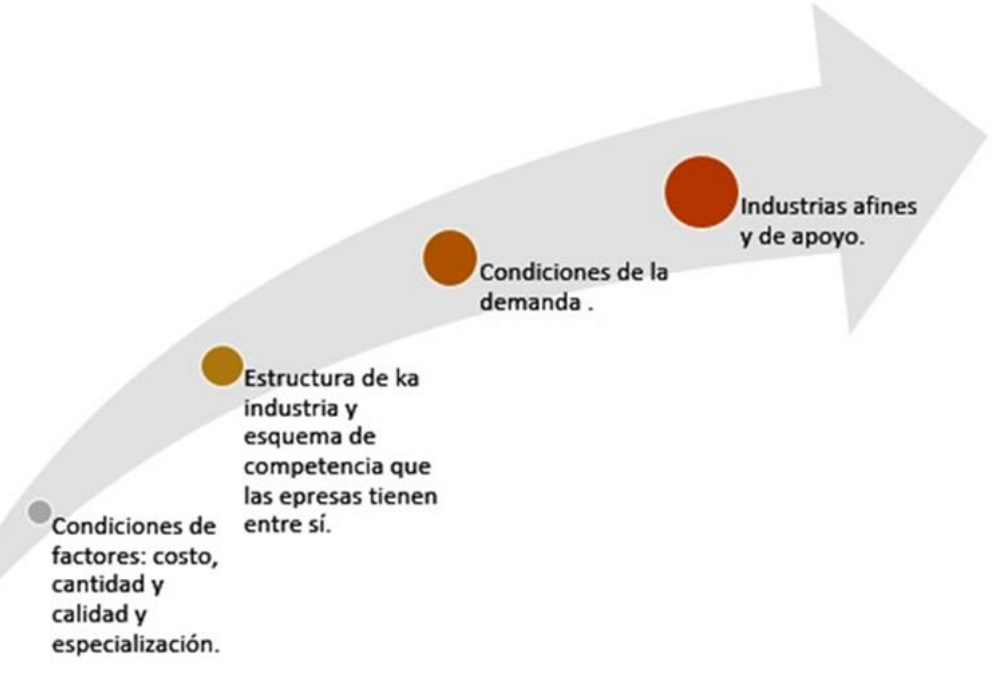

Fuente: Elaboración propia con base en Porter y Kramer (2006).

Según este mismo par de investigadores, existen tres formas de brindar valor para la empresa y para la sociedad que integran un ciclo a favor de todos, las cuales pueden ser observadas en la figura 7.

Figura 7. Formas para crear valor compartido.
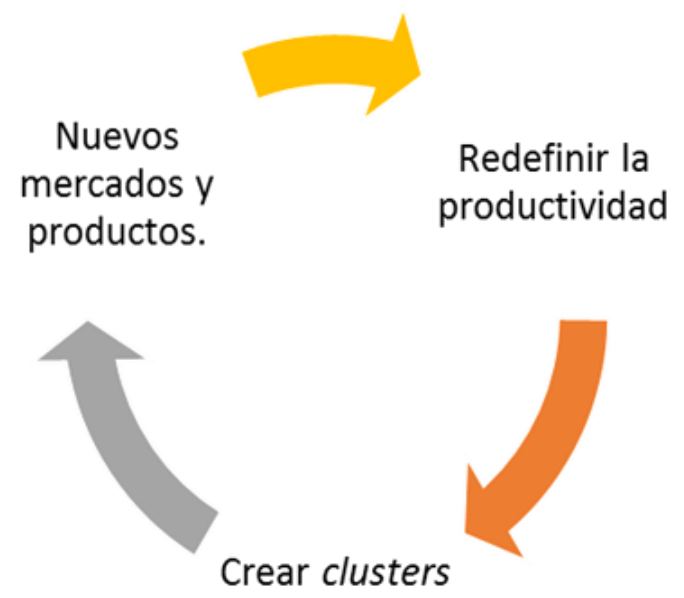

Fuente: Elaboración propia con base en Porter y Kramer (2011).

1) Nuevos mercados y productos. La búsqueda de otras alternativas que permitan destacar y abordar a otros clientes.

2) Redefiniendo la productividad en la cadena de valor. Poder modificar actividades de la empresa y su organización conforme lo requiera la demanda y el mercado. 


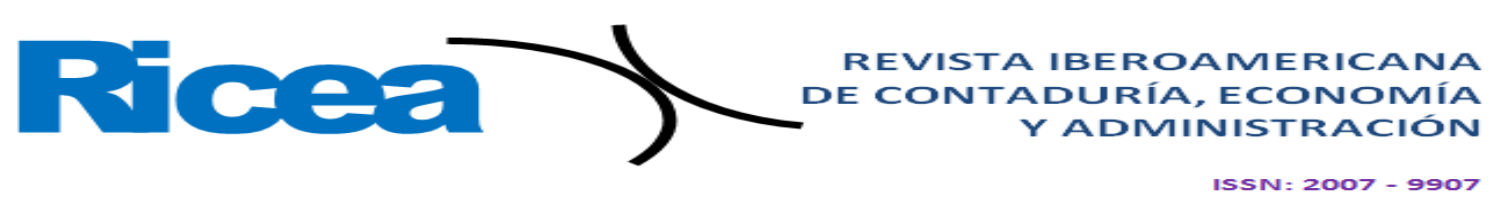

3) Crear clusters. Son grupos locales de apoyo a la empresa para crear potenciales específicos (Porter y Kramer, 2011).

Todo esto para encontrar soluciones a los problemas sociales con estrategias empresariales. Las organizaciones deben liderar para poder crear un valor económico que contribuya a la empresa misma y a la sociedad. Es decir, para que exista un valor compartido empresarialmente, se requiere de los factores organizados en la figura 8.

Figura 8. Factores principales del valor compartido.

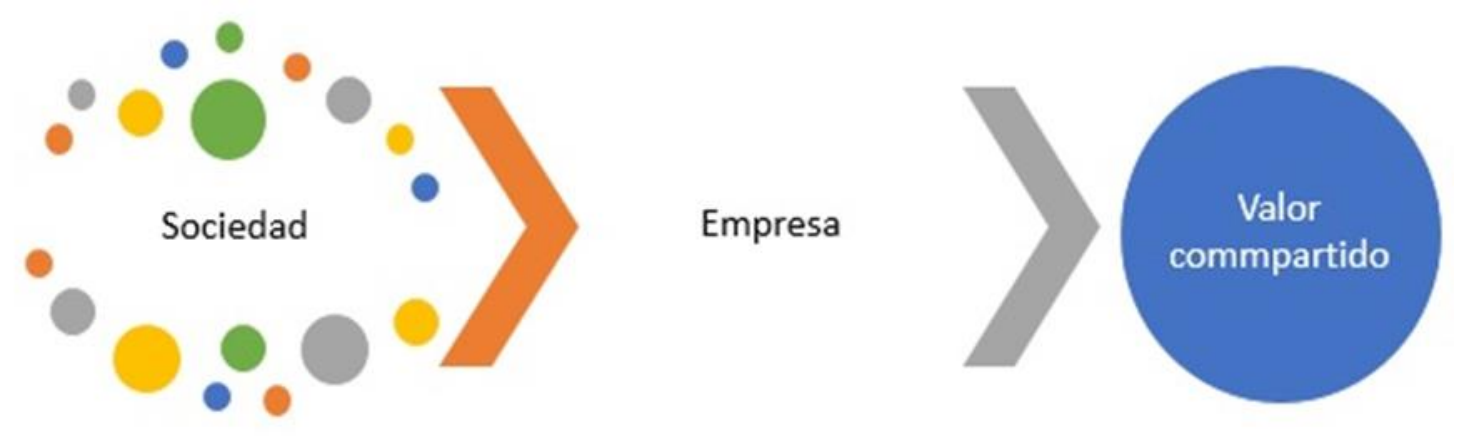

Fuente: Elaboración propia con base en Porter y Kramer (2011).

Dichos factores permiten trabajar en conjunto para crear el valor económico y el valor para la sociedad, atendiendo tanto sus necesidades como sus desafíos. Sin duda las empresas tienen que fusionar el progreso social con el éxito del negocio. Para comprender aún más el concepto de valor compartido, Porter y Kramer (2011) mencionan que se debe exhortar a los líderes y ejecutivos para que desarrollen nuevas habilidades y conocimientos, con una visión mucho más profunda de lo que la sociedad requiere para satisfacer sus necesidades, además de la concientización de cuáles son las verdaderas bases de productividad de la organización y la capacidad de colaborar entre sujetos, ya sea con fines o no lucrativos.

Un sector de enlace debería ser el Gobierno; debe regular leyes que permitan que el valor compartido sea aplicado, y la mejor forma de hacerlo es redefiniendo el concepto de organización, no considerándolo solo como la creación de utilidades, esto con la finalidad de lograr no solo un crecimiento en la economía local, sino en la productividad dentro de la economía global para darle una nueva forma al capitalismo y su relación con la sociedad. 


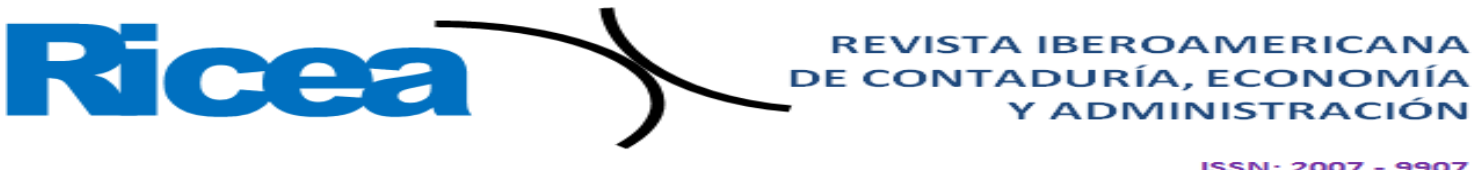

Porter y Kramer (2011) dejan en claro que cambiar los objetivos y descartar las estrategias a corto plazo son la nueva fórmula para generar una nueva alianza entre el mercado y la sociedad. Y el Gobierno, además, puede participar con la promoción de grupos de apoyo en donde se incorporen otros valores económicos, con la finalidad de optimizar el principal objetivo de las empresas y corporaciones, que es el lucro con el bien social y bien común.

El cambio más drástico para las organizaciones es el reconocerse como una empresa con valor social compartido y una empresa responsable es aquella que sigue el modelo de $R S C$ (Responsabilidad Social Corporativa). Una empresa de este segundo tipo RSC es aquella que renueva sus decisiones de manera que se conjunten los actores que la rodean y que va más allá de una situación económica, es decir es consciente en aspectos sociales y medioambiente, así como finanzas claras y miden el impacto de sus acciones de manera periódica.

En lo que respecta a este tipo de responsabilidades, integran y reconocen las preocupaciones sociales y medioambientales con el propósito de satisfacerlas y mostrar un enfoque basados en los valores éticos. Mientras que la creación de valor compartido pretende que las empresas se comprometan realmente con la sociedad y, con ello, obtener resultados conjuntos.

Para el año 2012, mundialmente diferentes empresas operaban programas del modelo RSC. En la tabla 5 se enlistan unos cuantos ejemplos de organizaciones de este tipo en México.

Tabla 5. Empresas con RSC en México.

\begin{tabular}{|l|l|}
\hline \multicolumn{1}{|c|}{ Empresa } & \multicolumn{1}{c|}{ RSC (Responsabilidad Social Corporativa) } \\
\hline GRUPO BIMBO & $\begin{array}{l}\text { Generar acciones que colaboren energéticamente en los retos venideros de la } \\
\text { sociedad. }\end{array}$ \\
\hline CEMEX & $\begin{array}{l}\text { Comprometido en los momentos difíciles con la sociedad, e interesado en los } \\
\text { aspectos sociales y de salud. }\end{array}$ \\
\hline COCA-COLA & $\begin{array}{l}\text { Contribuye con acciones caritativas, y busca el bienestar individual, social y } \\
\text { ambiental. }\end{array}$ \\
\hline GRUPO HERDEZ & $\begin{array}{l}\text { Empresa que tiene una fortaleza especialmente en las donaciones } \\
\text { filantrópicas. }\end{array}$ \\
\hline
\end{tabular}

Fuente: Elaboración propia.

Dichas empresas se incluyen en este listado con base en tres dimensiones, que son la gestión económica, social y medioambiental. Para ellas, ser una empresa RSC incluye algo más que seguir un modelo administrativo al pie de la letra. En ese sentido, es muy importante considerar la posición y postura nacional, en este caso de México. Para poder fortalecerse se necesitan factores como crecimiento, competitividad y sostenibilidad, sin embargo, el reto también es cultural, debido a que 


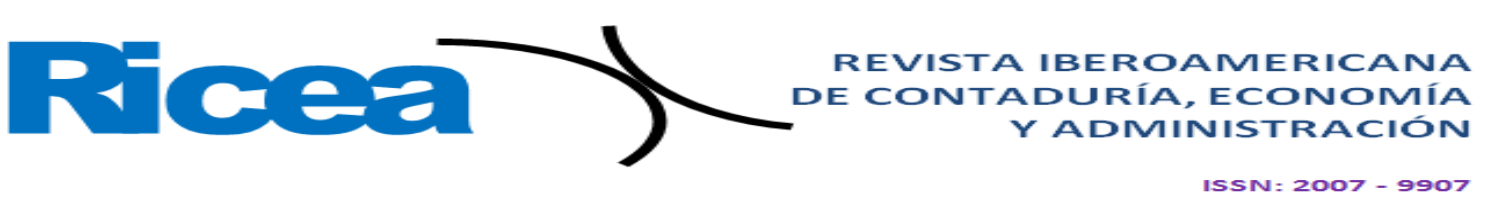

Figura 10. Representación gráfica de la teoría general de sistemas (TGS).

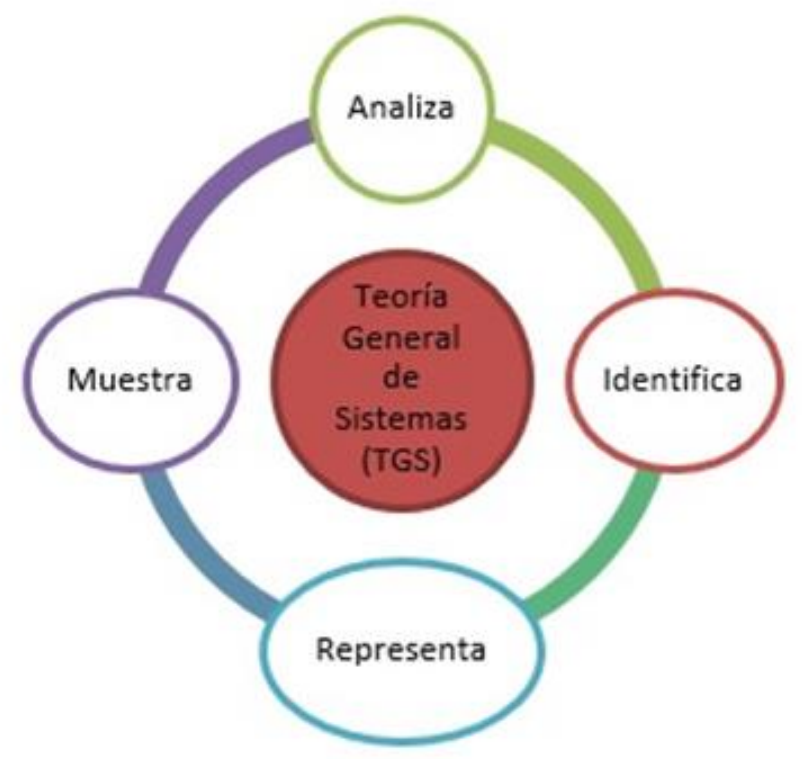

Fuente: Elaboración propia con base en Gutiérrez (2005).

Dado que el tema de la TGS es la formulación y derivación de aquellos principios que son válidos para todos los "sistemas" en general, un ejemplo en donde es posible apreciar esto es en la física, ya que esta ciencia se ocupa de sistemas de diferentes niveles de generalidad: se dilata desde sistemas bastante especiales hasta leyes especiales de disciplinas físicas, como la mecánica o la óptica. Se diría, entonces, que una teoría general de los sistemas sería un instrumento útil al dar, por una parte, modelos utilizables y transferibles entre diferentes campos y evitar, por otra, vagas analogías que a menudo han perjudicado el progreso en dichos campos (Von Bertalanffy, 1976).

En contraparte, se estipula que la TGS debe interpretarse como una ciencia de los sistemas en el sentido específico de doctrina de principios aplicables a todos los sistemas (o a subclases definidas de ellos). Rigurosamente desarrollada, deberá exhibir una estructura axiomática en la que aparezcan definiciones precisas y axiomas adecuados, a partir de los cuales se hagan deducciones a priori (Hidalgo, 1978). La TGS, a través del análisis de las totalidades y las interacciones internas de estas y las externas con su medio, es, ya en la actualidad, una poderosa herramienta que permite la explicación de los fenómenos que se suceden en la realidad, y también hace posible la predicción de la conducta futura de esa realidad.

Es un enfoque que debe utilizar el científico, ya que su rol, a nuestro juicio, es, justamente, el conocimiento y la explicación de la realidad, o de una parte de ella (sistemas), en relación con el medio que la rodea, $\mathrm{y}$, sobre la base de esos conocimientos, poder predecir el comportamiento de 


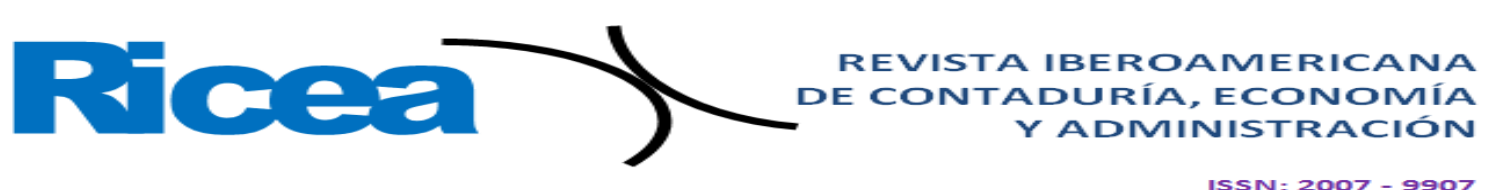

esa realidad, dadas ciertas variaciones del medio o entorno en el cual se encuentra inserta (Johansen, 1993).

Existe otro aspecto aún más importante de la TGS. Puede parafrasearse mediante una feliz formulación debida al bien conocido matemático y fundador de la teoría de la información, Warren Weaver, según Von Bertalanffy (1976).

La física clásica, dijo este, tuvo gran éxito al desarrollar la teoría de la complejidad no organizada. Por ejemplo, el comportamiento de un gas es el resultado de los movimientos desorganizados, e imposibles de seguir aisladamente, de innumerables moléculas; en conjunto, lo rigen las leyes de la termodinámica. La teoría de la complejidad no organizada se arraiga a fin de cuentas en las leyes del azar y la probabilidad y en la segunda ley de la termodinámica. En contraste, hoy el problema fundamental es el de la complejidad organizada. Conceptos como los de organización, totalidad, directiva, teleología y diferenciación son ajenos a la física habitual. Sin embargo, asoman a cada paso en las ciencias biológicas, del comportamiento y sociales, y son de veras indispensables para vérselas con organismos vivientes o grupos sociales. De esta manera, un problema fundamental planteado a la ciencia moderna es el de una teoría general de la organización. La teoría general de los sistemas es capaz en principio de dar definiciones exactas de semejantes conceptos y, en casos apropiados, de someterlos a análisis cuantitativo (Von Bertalanffy, 1976).

\section{Definición de sistema}

Acorde con la TGS, un sistema se describe como un conjunto de elementos que interactúan y tienen un objetivo común. Todo sistema está integrado por objetos o unidades agrupadas de tal manera que constituyan un todo lógico y funcional que es mayor que la suma de esas unidades.

Por otra parte, se tienen algunas definiciones complementarias:

- $\quad$ Parte de un universo, con una extensión limitada en espacio y tiempo.

- Un conjunto de objetos relacionados entre sí mismos y entre sus atributos.

- $\quad$ Es la estructura u organización, donde se muestran claramente las relaciones entre sus partes.

- Es un conjunto de entidades caracterizadas por ciertos atributos, que tienen 


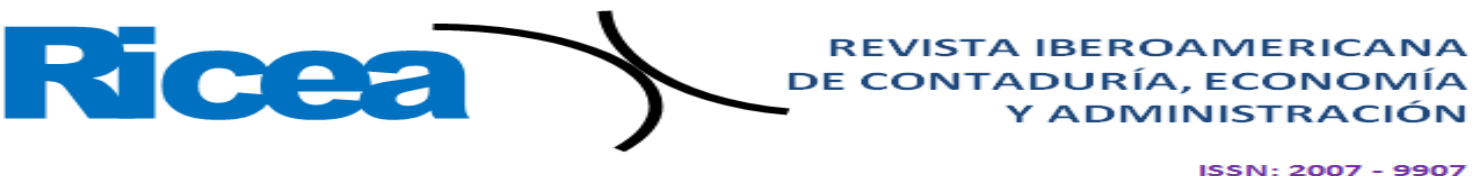

relaciones entre sí localizadas en un cierto medio ambiente, con un cierto objetivo (Trasobares, 2003).

- Otra definición de sistema basada en la TGS es aquella que lo describe como un conjunto de partes relacionadas para unificarse.

Los sistemas se dividen en dos: los cerrados y abiertos, tal como se ilustra en la figura 11 (Robbins y Coulter, 2010). Adicionalmente, un sistema es un conjunto de partes que tienen relación entre sí para que al final se junten y realicen una cierta función.

Figura 11. Representación de tipos de sistemas.

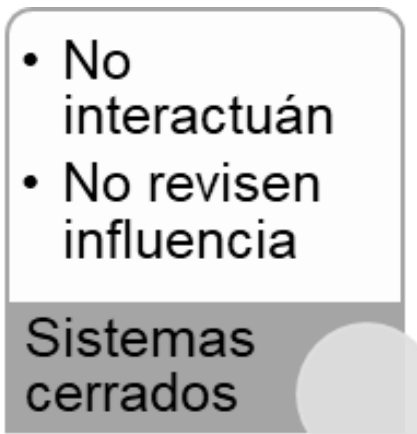

- Influenciados - Interactuán

Fuente: Robbins y Coulter (2010).

Por otro lado, contrario al entender de Robbins y Coulter (2010), quienes, como hemos visto, dividen los sistemas en cerrados y abiertos, existen otros autores que afirman que no hay sistema totalmente cerrado ni abierto, tal y como lo indica Hernández (2011).

Según Hernández (2011), los sistemas dependen de su composición material, objetivos de sus elementos y su capacidad. Y se dividen en abstracto y concreto, tal como se observa en la figura 12.

Figura 12. Tipos de sistemas de acuerdo a Hernández (2011).

\section{Abstracto}

Concreto

Fuente: Hernández (2011) 


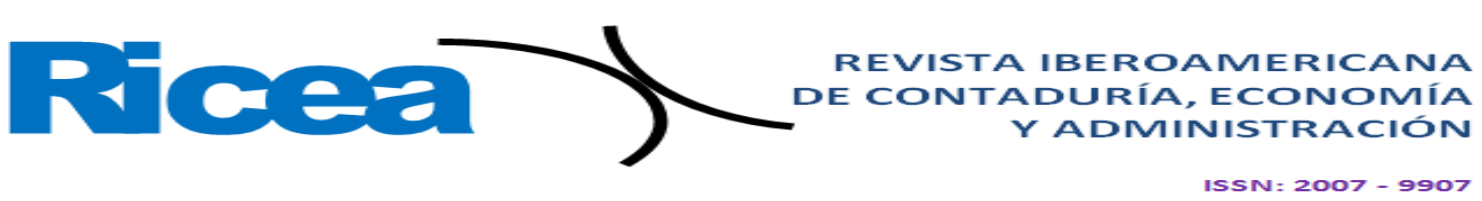

Por lo anterior, el proceso y fases de la investigación cualitativa y el diseño de investigación sitúa al investigador en el mundo empírico y determina las actividades que tendrá que realizar para poder alcanzar el objetivo propuesto.

Además, la investigación cualitativa establece que observadores competentes y cualificados pueden informar con objetividad, claridad y precisión acerca de sus propias observaciones del mundo social, así como de las experiencias de los demás.

Las cuatro fases fundamentales en el proceso de investigación cualitativa del proyecto son, según Rodríguez et al. (1996), las siguientes:
1) preparatoria,
2) trabajo de campo,
3) analítica e
4) informativa.

\section{Resultados y discusión}

El objetivo principal de esta investigación es la vinculación académica con el sector productivo de la región del Valle de Teotihuacán mediante un análisis de las condiciones económicas de los productores y comercializadores del nopal. Así, se recopila información basada en las condiciones laborales, proceso productivo, formas de comercialización la relación costobeneficio y la valoración de la cobertura de las hectáreas sembradas contra las cosechadas. Este estudio se enfocó en siete municipios dedicados a la producción del nopal en el Estado de México: Acolman, Axapusco, Nopaltepec, Otumba, San Martín De Las Pirámides, Temascalapa y Teotihuacán. Estos se ubican en la región nororiente del Estado de México.

Cabe mencionar que esta investigación se desarrolló por un grupo multidisciplinario; sus integrantes son investigadores de las áreas de economía, administración, contabilidad e informática adscritos al Centro Universitario UAEM Valle Teotihuacán y Valle de México.

Así, atendiendo el compromiso social del Centro Universitario UAEM Valle de Teotihuacán, se considera trascendental la vinculación académica con el sector productivo. Derivado de la investigación realizada, el presente proyecto corresponde a la Línea de Generación y Aplicación del Conocimiento (LGAC) de Administración Contable y Financiera en la Empresa, como parte del fortalecimiento del cuerpo académico Administración Contable-Financiera del 


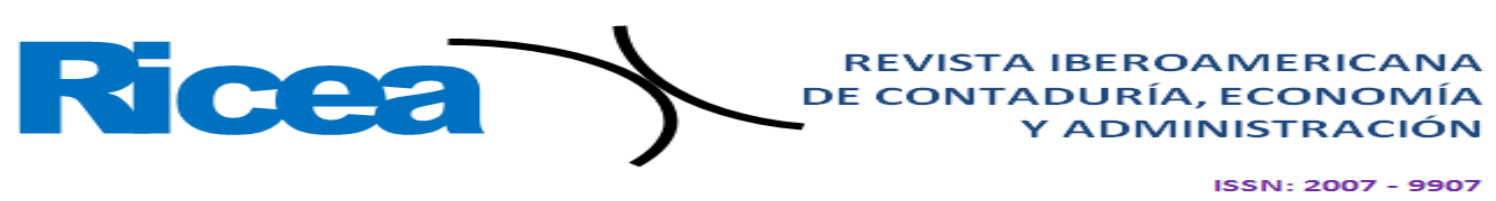

fumigadores afectan severamente los plantíos. Esto influye en el desinterés de los productores para implementar nuevas alternativas que generen mejores condiciones de producción, comercialización y utilidades. También se identifica que la producción de la tuna es únicamente por temporada, lo que causa que algunos productores y comercializadores acaparen el acopio excesivo de los productores más pequeños, con lo que establecen precios bajos de compra, pero altos en su venta. Por último, este proyecto tiene como prioridad incentivar y capacitar a los productores para obtener un mejor beneficio, ya que la mayoría de ellos que se dedican al cultivo son de edad avanzada y con estudios relativamente limitados, mientras que a las nuevas generaciones de jóvenes no les resulta interesante la actividad del cultivo del nopal por las mínimas o nulas ganancias que se obtienen.

\section{Conclusiones}

La región del Valle de Teotihuacán se identifica como una zona productora agrícola de nopal tunero, verdulero y xoconostle, no obstante, los incentivos para incrementar la producción son casi nulos. Una problemática identificada es que los productores no consideran benéfico un periodo con abundante cosecha, puesto que, derivado de una excesiva demanda de producto, los precios se abaratan por debajo de los costos de producción, a tal grado de que no resulta redituable para los productores.

El ciclo de vida del producto es muy corto en los tres productos aquí contemplados. Y aunque un reducido porcentaje de productores produce derivados del nopal, mermelada, dulce y escabeche, son los principales productos, el resto de productores considera que la falta de capital para infraestructura y el desconocimiento para emprender derivados del nopal son limitantes, lo que redunda en una falta de incentivos para aprovechar la cosecha, inclusive en ocasiones prefieren no recolectarlo.

Otro factor que limita su expansión en el mercado es que solo $10 \%$ de los productores está registrado ante la Secretaría de Hacienda y Crédito Público (SHCP); el resto vende su producción a intermediarios a precios bajos, sin tener opciones de venta y sin posibilidad de obtener beneficios a través de los programas gubernamentales destinados a las actividades agrícolas.

Es prioritario impulsar el campo a través de políticas orientadas y focalizadas a actividades redituables. Una opción que es necesario considerar es proteger la agroindustria nacional; en el mercado nacional se observan productos del nopal producidos en otros países. Incentivar y 


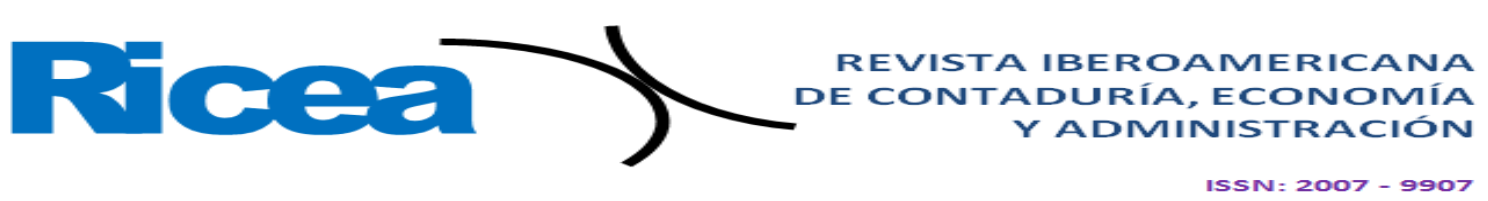

capacitar a los productores es importante, ya que la mayoría de ellos son de edad avanzada y a los jóvenes no les parece atractiva la actividad por las mínimas o nulas ganancias que obtienen.

\section{Agradecimientos}

Los autores agradecen a la Secretaría de Investigación y Estudios Avanzados (SIyEA) de la UAEM por las facilidades otorgadas para realizar la presente investigación. Además, se agradece a los siete municipios principales del Estado de México: Acolman, Axapusco, Nopaltepec, Otumba, San Martín de las Pirámides, Temascalapa y Teotihuacán, que aportaron datos estadísticos e información valiosa a esta investigación. Finalmente, esta investigación consolida los vínculos académicos entre los Centros Universitarios de Valle de Teotihuacán y de Valle de México de la UAEM con el Tecnológico de Estudios Superiores de Coacalco.

\section{Referencias}

Aguilar, A. (2003). Guía para la producción nopal tunero en el Estado de México. Estado de México, México: Secretaría de Agricultura y Desarrollo Rural. Recuperado de http://biblioteca.inifap.gob.mx:8080/jspui/handle/123456789/1745.

Aranda, Ó. S. (16 de septiembre de 2014). Más que un símbolo mexicano: el nopal. Relatos de la naturaleza. Recuperado de https://relatosdelanaturaleza.org/2014/09/16/mas-que-unsimbolo-mexicano-el-nopal/.

Arnold, M. y Osorio, F. (1998). Introducción a los conceptos básicos de la Teoría General de Sistemas. Cinta Moebio: Revista Electrónica de Epistemología de Ciencias Sociales, 3(1), 40-49.

Cometuna, Red Nopal y Conabio (2009). Nopales, tunas y xoconostles Consejo Mexicano de Nopal y Tuna, A.C., Red Nopal y Comisión Nacional para el conocimiento y Uso de la Biodiversidad. $\quad 2^{\mathrm{a}}$ edición, México. Recuperado de http://200.12.166.51/janium/Documentos/6579.pdf.

Comisión Nacional para el Conocimiento y Uso de la Biodiversidad [Conabio]. (2014). Nopales Producción e innovación. México: Comisión Nacional para el Conocimiento y Uso de la Biodiversidad. $\quad$ https://www.gob.mx/conabio

https://sites.google.com/site/nopal2c2018/home/produccion-nopal 


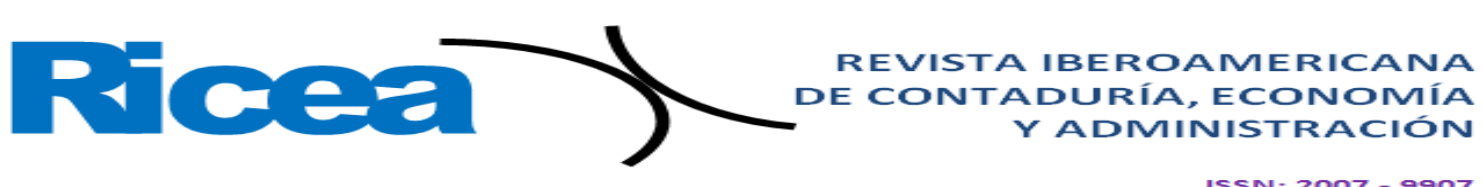

Mandujano, A., Morales, J., Herrera, H., Corona, L. E. y Juárez, J. M. (2018). Evaluación del comportamiento electroquímico del extracto de nopal (Opuntia Ficus-Indica) como posible inhibidor de corrosión. Revista de Metalurgia, 4(53), 1-10.

Millán, H. y Pérez, R. (2008). Desigualdad social y pobreza en el Estado de México: ¿convergencia o divergencia? Revista Economía y Sociedad, 21(15), 17-39. Recuperado de https://www.redalyc.org/articulo.oa?id=51002102.

Mutis, G. (18 de junio de 2013). Valor compartido, una estrategia empresarial de alto impacto. El Espectador. Recuperado de http://www.elespectador.com/publicaciones/especial/articulo428561-valor-compartido-una-estrategia-empresarial-de-alto-impacto.

OMPI, Organización Mundial de la Propiedad Intelectual (2018). Guía sobre los aspectos de propiedad intelectual relacionados con los acuerdos de acceso y participación en los beneficios. $1-98 . \quad$ Recuperado de https://www.wipo.int/edocs/pubdocs/es/wipo_pub_1052.pdf.

Porter, M. E. and Kramer M. R. (2006). Strategy and Society: the link between competitive advantage and Corporate Social Responsibility. Harvard Business Review, 12(84), 78-92. Retrieved from https://www.sharedvalue.org/sites/default/files/resourcefiles/Strategy_and_Society.pdf.

Porter, M. E. and Kramer, M. R. (2011). Creating Shared Value. How to reinvent capitalism and unleash a wave of innovation and growth. Harvard Business Review, 12(89), 62-77. Retrieved from https://hbr.org/2011/01/the-big-idea-creating-shared-value.

Robbins, S. P. y Coulter, M. (2010). Administración. Estado de México, México: Pearson Educación.

Rodríguez, G, Gil, J. y García, E. (1996). Metodología de la investigación cualitativa. Málaga, España: Editorial Aljibe.

Secretaría de Economía. (2015). Información económica y estatal. Estado de México. México: Secretaría de Economía. Recuperado de https://www.gob.mx/cms/uploads/attachment/file/43334/Estado_de_Mexico.pdf.

Servicio de Información Agroalimentaria y Pesquera [SIAP] (2016). Estadística de producción agrícola. Gobierno de México. Recuperado de http://infosiap.siap.gob.mx/gobmx/datosAbiertos.php 


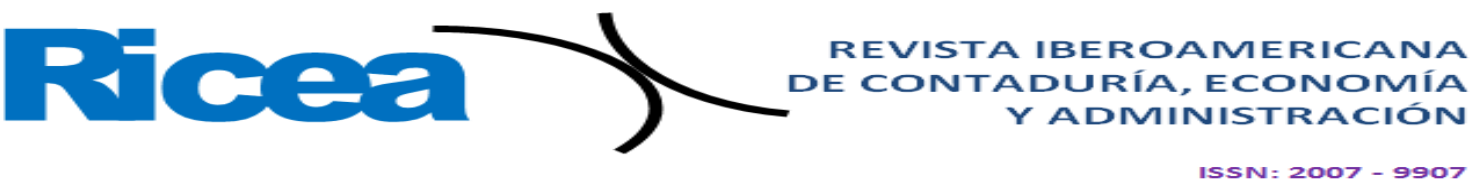

Trasobares, A. (2003). Los sistemas de Información: Evolución y Desarrollo. Proyecto Social: Revista de Relaciones Laborales, (10-11), 149-165. Recuperado de https://dialnet.unirioja.es/servlet/articulo?codigo=793097.

Von Bertalanffy, L. (1976). Teoría general de los sistemas: Fundamentos, desarrollo, aplicaciones. México: Fondo de Cultura Económica. 


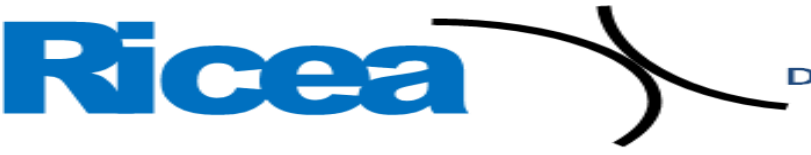

REVISTA IBEROAMERICANA DE CONTADURÍA, ECONOMÍA

\begin{tabular}{|l|l|}
\hline Rol de Contribución & Autor (es) \\
\hline Conceptualización & Blanca Estela Hernández Bonilla \\
\hline Metodología & Blanca Estela Hernández Bonilla \\
\hline Software & NO HAY CONTRIBUCIÓN \\
\hline Validación & $\begin{array}{l}\text { Blanca Estela Hernández Bonilla (principal) Adriana } \\
\text { Mercedes Ruiz Reynoso (que apoya) }\end{array}$ \\
\hline Análisis Formal & $\begin{array}{l}\text { Blanca Estela Hernández Bonilla (principal) Verónica } \\
\text { Ramírez Cortés (que apoya) Sendy Janet Sandoval Trujillo } \\
\text { (que apoya) Margarita Dávila Hernández (que apoya) } \\
\text { Adriana Mercedes Ruiz Reynoso (que apoya) }\end{array}$ \\
\hline Investigación & $\begin{array}{l}\text { Verónica Ramírez Cortés (principal) Sendy Janet Sandoval } \\
\text { Trujillo (que apoya) Margarita Dávila Hernández (que } \\
\text { apoya) }\end{array}$ \\
\hline Recursos & Blanca Estela Hernández Bonilla \\
\hline Curación de datos & Adriana Mercedes Ruiz Reynoso \\
\hline $\begin{array}{l}\text { Escritura - Preparación del } \\
\text { borrador original }\end{array}$ & Adriana Mercedes Ruiz Reynoso \\
\hline $\begin{array}{l}\text { Escritura - Revisión y } \\
\text { edición }\end{array}$ & $\begin{array}{l}\text { Adriana Mercedes Ruiz Reynoso (principal) Blanca Estela } \\
\text { Hernández Bonilla (que apoya) }\end{array}$ \\
\hline Visualización & $\begin{array}{l}\text { Adriana Mercedes Ruiz Reynoso (principal) Blanca Estela } \\
\text { Hernández Bonilla (que apoya) }\end{array}$ \\
\hline Supervisión & $\begin{array}{l}\text { Adriana Mercedes Ruiz Reynoso (principal) Blanca Estela } \\
\text { Hernández Bonilla (que apoya) }\end{array}$ \\
\hline $\begin{array}{l}\text { Administración de } \\
\text { Proyectos }\end{array}$ & $\begin{array}{l}\text { Adriana Mercedes Ruiz Reynoso (que apoya) Blanca Estela } \\
\text { Hernández Bonilla (principal) }\end{array}$ \\
\hline Adquisición de fondos & Blanca Estela Hernández Bonilla \\
\hline
\end{tabular}

